\title{
Atypical Presentation of Capillary Hemangioma of Upper Eyelid: A Case Report
}

\author{
Jawed Iqbal $^{1}$ and Arjun Chaudhary ${ }^{2}$ \\ ${ }^{1}$ Senior Resident (Department of Ophthalmology, Anugrah Narayan Magadh Medical College and Hospital, \\ Gaya, 823001) \\ ${ }^{2}$ Professor (Department of Ophthalmology, Anugrah Narayan Magadh Medical College and Hospital, Gaya,
} 823001)

\begin{abstract}
Capillary hemangioma is a benign vascular tumour composed of proliferating endothelial cells within fibrous tissue. Other names for this tumour include "benign hemangioendothelioma" and "strawberry nevus." It is the most common tumour of infancy, occurring in 1 to 2 per cent of infants with a female predilection. Capillary hemangioma is a hamartomatous localized proliferation of vascular tissue in a normal location. They manifest by 18 months of age. Most hemangiomas regress spontaneously by age of seven years. We reported a female child of 7 years with gradually increasing mass over upper eyelid since 3 months that was found to be capillary haemangioma on histopathological examination. Atypical late presentation of capillary hemangioma of right upper eyelid is presented in which meticulous surgical excision provided excellent results. Recurrence after surgical excision can occur in capillary hemangiomas. Steroids, laser and radiotherapy are some other treatment options.
\end{abstract}

Keywords: Capillary hemangioma, Eyelid tumour, hamartomas

\section{Introduction}

Periocular capillary hemangiomas are common benign vascular tumours seen in children. Histologically, they are characterized by proliferating endothelial cells. They generally present within a few weeks after birth and undergo a rapid proliferating phase, followed by a period of relative quiescence, regressing after a few years into an involution phase $(1,2)$. The clinical course can be variable. Demographically, these cases occur commonly among females and have not been reported to vary by race, ethnicity, or geographic location $(3,4)$.

Acceptable indications for intervention may include rapidly enlarging lesions, obstruction of the visual axis, significant induced astigmatism, and cosmetic concerns. Here, a case study is presented, along with some of the current treatment modalities. The modalities available are intralesional and systemic steroid, bleomycin, interferon-alpha, vincristine, cyclophosphamide, topical timolol maleate, oral propranolol, and surgical excision $(1,5)$.

\section{Case Report}

History of present illness: A 7 year old female, presented with painless, progressive mass of right upper eyelid slightly lateral to the midline, which was gradually increasing in size since 3 months. Patient was not having any other complaints apart from that. There was no significant medical, surgical, family and birth history. Child was fully immunized as per age. General and systemic examination of the patient was normal. Visual acuity of the patient was $6 / 6$ in both eyes. Left eye findings were within normal limits. Local examination of right upper eyelid showed a reddish colour lobulated, movable, non tender mass $1.5 \mathrm{~cm}$ in height, width and depth firm in consistency (Fig. 1). There was no crusting, ulceration, bleeding over the mass. Rest of the anterior and posterior segment examination of right eye was normal.

Procedure: A differential diagnosis of pyogenic granuloma or hemangioma was made and total excision biopsy was carried out under general anaesthesia and sent for histopathological examination. The skin incision was then sutured with mersilk 6, 4 sutures were taken. Histopathological examination of excised lesion shows small to medium sized capillaries and fibrous tissue in the intervening stroma and confirmed diagnosis of capillary hemangioma. Patient was started antibiotic drops and ointment, anti-inflammatory and analgesics drugs post operatively and discharged.

Postoperative period: Follow up examination after 7 days was done. Patient was asymptomatic and there was no lid oedema and no discharge. Sutures were removed on 7th post operative day (Fig. 2). 


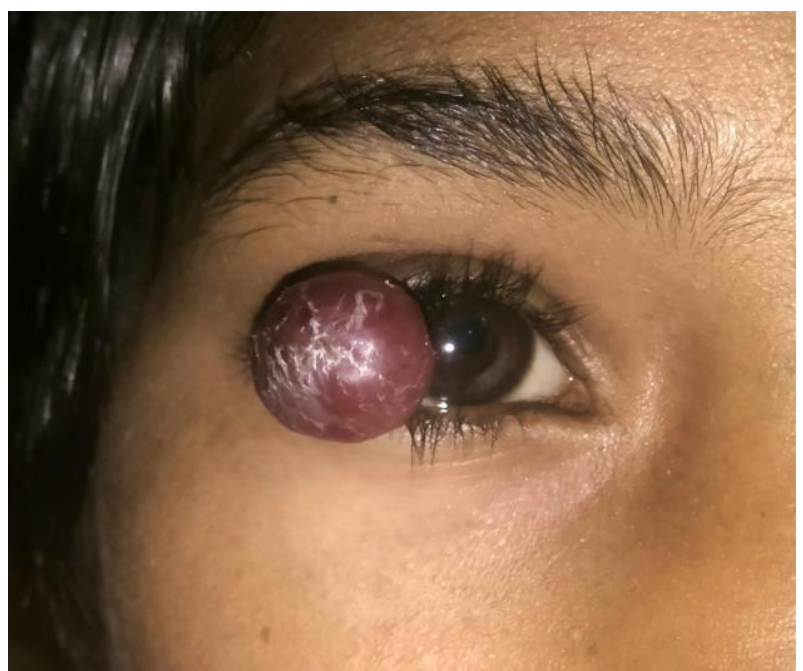

Fig. 1: A 7 year old female with capillary hemangioma on upper lateral side of eyelid

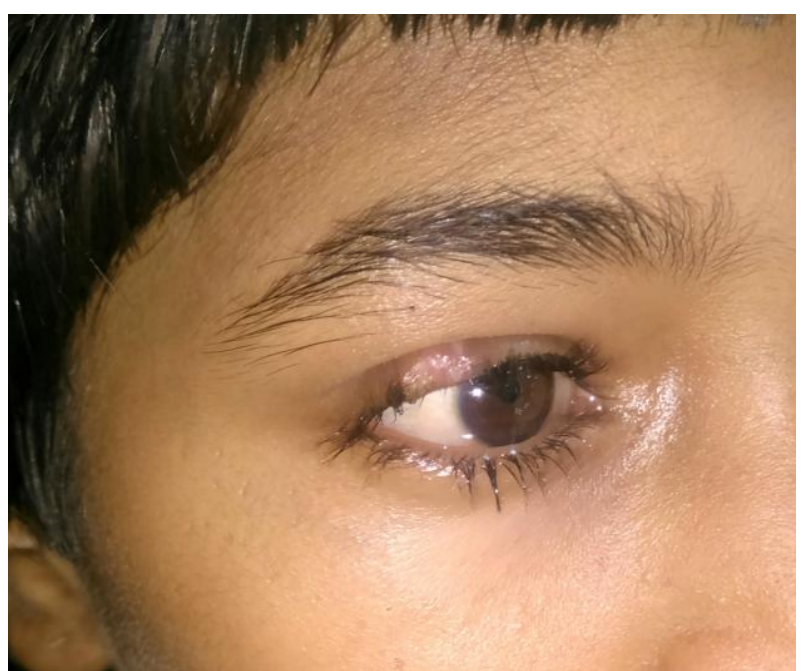

Fig. 2: Postoperative $\left(7^{\text {th }}\right.$ day $)$ view of the patient

\section{Discussion}

The capillary hemangioma, also known as a benign hemangioendothelioma, is a common vascular lesion of childhood, which occurs in 1-2 per cent of infants and is the most common orbital tumour found in children. Girls are more commonly affected than boys, with a 3:2 ratio (6). A periorbital hemangioma may appear as a superficial cutaneous lesion, subcutaneous lesion, deep orbital tumour, or combination of these types. Approximately one third of lesions are visible at birth, with the remainder manifest by 6 months of age. There is typically an initial rapid growth phase within 6 months of diagnosis, followed by a period of stabilization and subsequent involution over several years. It is estimated that approximately 75 per cent regress to some extent by the time the child reaches 7 years of age.

The history and presentation of a periorbital capillary hemangioma is so typical as to be diagnostic. Parents usually describes a growing red or purple spot that was not present at birth but becomes larger and thicker over the first few months of life. The lesion blanches with pressure, which is helpful in distinguishing it from the nonblanching 'portwine'. While most hemangiomas are not present at birth, more than half of them will manifest by 12 months of age and 90-100 per cent of the lesions will be present by 6-8 months of age. The peak size of the lesion is reached by 6-12 months, at which point the hemangioma, if left untreated, will remain stable for some time before beginning to involute. In fact, spontaneous involution is the rule for untreated capillary hemangiomas and progressive involution continues until 8 years of age. Forty per cent of hemangiomas completely regress by 4 years of age and 80 per cent completely regress by 8 years of age (7).

In most cases, capillary hemangiomas involute spontaneously without intervention and with good resulting cosmesis. On the other hand, visual axis obscuration, strabismus, optic nerve compression and proptosis, which may lead to exposure keratopathy, are all major indications for treatment (8). Systemic indications include thrombocytopenia and obstruction in oral and nasal passages. Early intervention is beneficial 
if significant anisometropia develops which may result in non treatable amblyopia. Local injection of $40 \mathrm{mg}$ triamcinolone and $6 \mathrm{mg}$ betamethasone sodium phosphate into the tumour may lead to involution in some cases. Large diffused tumours may be treated with alternate day administration of large doses of systemic steroids. Immunomodulator: Vincristine, Cyclophosphamide and Interferon alfa2a has been used as monotherapy or in combination with systemic corticosteroids. Surgical excision: Surgery carries the risks associated with general anesthesia. As the lesion is not encapsulated, surgical excision is often difficult and there is a risk of recurrence. Superficial radiotherapy (80-120 kV) may be given in a doses of 100-200 rad monthly for 6 months, with a total dose not exceeding 500-600 rad. Injection of sclerosing solutions is discouraged because of residual scarring (9). Systemic beta-blockers constitute a new and very promising treatment modality (8).

In our case, due to large size of capillary hemangioma, surgical approach was taken and hemangioma was successfully excised.

\section{Conclusion}

Though capillary hemangioma presents usually at birth and up to 1 year of age, it may present as late as 7 years. Meticulous surgical excision gives good results even in recurrent cases. Eyelid and orbital capillary hemangiomas are treatable vascular lesions. Ophthalmologists should be aware of the ocular complications of this tumour. Early recognition and timely diagnosis and treatment may prevent amblyopia and cosmetic disfigurement.

\section{References}

[1] Frank RC, Cowan BJ, Harrop AR, et al. Visual development in infants: Visual complications of periocular hemangiomas. J Plast Reconstr Aesthet Surg 2010;63:1-8.

[2] Tambe K, Munshi V, Dewsberry C, et al. Relationship of infantile periocular hemangioma depth to growth and regression pattern. J AAPOS. 2009;13:567-570.

[3] Wilson MW, Hoehn ME, Haik BG, et al. Low-dose cyclophosphamide and interferon alfa 2a for the treatment of capillary hemangioma of the orbit. Ophthalmology 2007;114:1007-1011.

[4] Elflein HM, Stoffelns BM, Pitz S. Laser and other treatment options in the therapy of infantile capillary eyelid and periorbital hemangiomas: An overview. Med Laser Appl 2010;25:242-249.

[5] Weiss AH, Kelly JP. Reappraisal of astigmatism induced by periocular capillary hemangioma and treatment with intralesional corticosteroid injection. Ophthalmology 2008;115:390-397.

[6] Yanoff M. 'Ophthalmology' 3rd edition 2009: 1426.

[7] Peralta RJ, Glavas. Review of Capillary Hemangioma. New York University and the Manhattan Eye, Ear and Throat Hospital, New York. Eye Net Magazine 2009

[8] Kanski JJ. Clinical Ophthalmology A Systemic Approach, 7th edition 2011; pp. 103

[9] Sihota and Tandon. 'Parson's diseases of eye'. 21st edition. 2015; pp.466. 